\title{
A pilot study using dynamic contrast enhanced-MRI as a response biomarker of the radioprotective effect of memantine in patients receiving whole brain radiotherapy
}

\author{
Philip Wong ${ }^{1}$, Ilana R. Leppert ${ }^{2}$, David Roberge ${ }^{1}, K_{\text {Karim Boudam }}{ }^{1}$, Paul D. Brown $^{3}$, \\ Thierry Muanza ${ }^{4}$, G. Bruce Pike ${ }^{2,5}$, Jeffrey Chankowsky ${ }^{6}$ and Catalin Mihalcioiu ${ }^{7}$ \\ ${ }^{1}$ Department of Radiation Oncology, Centre Hospitalier de L'Université de Montréal, Montréal, Québec, Canada \\ 2 Montreal Neurological Institute, McGill University, Montreal, Quebec, Canada \\ ${ }^{3}$ Department of Radiation Oncology, Division of Radiation Oncology, the University of Texas MD Anderson Cancer Center, \\ Houston, TX, United States of America \\ ${ }^{4}$ Department of Oncology, Jewish General Hospital, Montreal, Québec, Canada \\ ${ }^{5}$ Departments of Radiology and Clinical Neurosciences, Hotchkiss Brain Institute, University of Calgary, Alberta, Canada \\ ${ }^{6}$ Diagnostic Radiology, McGill University Health Center, Montréal, Québec, Canada \\ 7 Department of Oncology, McGill University Health Center, Montreal, Québec, Canada \\ Correspondence to: Philip Wong, email: Philip.wong.chum@ssss.gouv.qc.ca
}

Keywords: MRI, biomarker, imaging, radiotherapy, memantine

Received: May 09, $2016 \quad$ Accepted: May 17, $2016 \quad$ Published: May 28, 2016

\section{ABSTRACT}

Purpose: This pilot prospective study sought to determine whether dynamic contrast enhanced MRI (DCE-MRI) could be used as a clinical imaging biomarker of tissue toxicity from whole brain radiotherapy (WBRT).

Method: 14 patients who received WBRT were imaged using dynamic contrast enhanced DCE-MRI prior to and at 8-weeks, 16-weeks and 24-weeks after the initiation of WBRT. Twelve of the patients were also enrolled in the RTOG 0614 trial, which randomized patients to the use of placebo or memantine. After the unblinding of the treatments received by RTOG 0614 patients, DCE-MRI measures of tumor tissue and normal appearing white matter (NAWM) vascular permeability (Initial Area Under the Curve (AUC) Blood Adjusted) was analyzed. Cognitive, quality-of-life (QOL) assessment and blood samples were collected according to the patient's ability to tolerate the exams. Circulating endothelial cells (CEC) were measured using flow cytometry.

Results: Following WBRT, there was an increasing trend in the vascular permeability of tumors $(p=0.09)$ and NAWM $(p=0.06)$ with time. Memantine significantly $(p=0.01)$ reduced NAWM AUC changes following radiotherapy. Patients on memantine retained (COWA $p=0.03$ ) better cognitive functions than those on placebo. No association was observed between the level of CEC and DCE-MRI changes, time from radiotherapy or memantine use.

Conclusions: DCE-MRI can detect vascular damage secondary to WBRT. Our data suggests that memantine reduces WBRT-induced brain vasculature damages.

\section{INTRODUCTION}

The incidence of brain metastasis in cancer patients has been $30-40 \%$ [1-3]. This figure may rise as novel therapeutic agents improve the systemic control of cancers outside of the central nervous system. The brain represents a watershed area for tumor metastasis to seed and colonize as the normal blood-brain-barrier (BBB) prevents systemic agents from reaching tumor cells. Radiotherapy currently remains as the main treatment option for patients with 
Table 1: Characteristics of the study patients

\begin{tabular}{|c|c|c|c|c|c|c|c|}
\hline Pt \# & $\begin{array}{l}\text { Primary } \\
\text { disease }\end{array}$ & $\begin{array}{ll}\text { Age } & \text { at } \\
\text { time } & \text { of } \\
\text { WBRT }\end{array}$ & $\begin{array}{l}\text { Number } \\
\text { of brain } \\
\text { lesions }\end{array}$ & $\begin{array}{l}\text { Volume } \\
\text { of tumor } \\
\text { analyzed } \\
\text { (cc) }\end{array}$ & \begin{tabular}{|l} 
Time between \\
WBRT and prior \\
surgery or SRS \\
brain treatment
\end{tabular} & $\begin{array}{l}\text { OS from } \\
\text { WBRT } \\
\text { (months) }\end{array}$ & $\begin{array}{l}\text { Use of } \\
\text { Pla, Mem } \\
\text { or Neither }\end{array}$ \\
\hline 1 & Breast & 34 & 1 & 1.73 & Sx: 4 weeks & 20.5 & Pla \\
\hline 2 & Breast & 53 & 4 & 0.78 & $\mathrm{~N} / \mathrm{A}$ & 33.6 & Pla \\
\hline 3 & Lung & 71 & 2 & 0.62 & $\begin{array}{l}\text { Sx: } 6 \text { weeks } \\
\text { RS: } 4 \text { weeks }\end{array}$ & 23.4 & Neither \\
\hline 4 & Breast & 51 & 1 & 1.58 & $\begin{array}{l}\text { Sx: } 6 \text { weeks } \\
\text { RS: } 4 \text { weeks }\end{array}$ & 18.6 & Mem \\
\hline 5 & Colon & 66 & 4 & 0.48 & RS: 4 weeks & 4.0 & Mem \\
\hline 6 & Lung & 65 & $>10$ & 0.94 & $\mathrm{~N} / \mathrm{A}$ & 5.6 & Mem \\
\hline 7 & Lung & 66 & 4 & 13.06 & N/A & 10.9 & Mem \\
\hline 8 & Melanoma & 65 & 3 & 0.53 & RS: 5 weeks & 4.9 & Mem \\
\hline 9 & Thryoid & 57 & 2 & 1.78 & RS: 59 weeks & 21 & Neither \\
\hline 10 & Lung & 67 & 6 & 0.60 & $\mathrm{~N} / \mathrm{A}$ & 7.2 & Plac \\
\hline 11 & Lung & 44 & 1 & 3.70 & RS: 2 weeks & 0 & Mem \\
\hline 12 & \begin{tabular}{|l|} 
Lung \\
\end{tabular} & 78 & $>10$ & 0.62 & $\mathrm{~N} / \mathrm{A}$ & 5.9 & Plac \\
\hline 13 & Lung & 63 & 2 & 1.37 & $\begin{array}{l}\text { Sx: } 5 \text { weeks } \\
\text { RS: } 2 \text { weeks }\end{array}$ & 28.5 & Mem \\
\hline 14 & Breast & 57 & 2 & 0.65 & RS: 44 weeks & 8.6 & Plac \\
\hline
\end{tabular}

WBRT: Whole brain radiotherapy; Sx: Surgery; RS: Radiosurgery; N/A: no surgery or RS; OS: Overall Survival; Pla: Placebo; Mem: Memantine

brain metastases [4].

Despite the increasing use of radiosurgery to treat brain metastases, patients with brain metastases often receive whole brain irradiation (WBRT) for widespread metastases, post-radiosurgery progression or leptomeningeal seeding [4]. WBRT may control the progression of brain metastases and lengthen lifespan, but it may induce cognitive deficits. As radiation may induce neuronal N-methy-D-aspartate (NMDA) receptor stimulation and excitotoxicity, inhibition of the NMDA receptor using a competitive antagonist, memantine may reduce WBRT-induced cognitive decline. The Radiation Therapy Oncology Group (RTOG) 0614 study randomized patients treated with WBRT to receive concurrent placebo or memantine [5]. The RTOG 0614 study demonstrated that patients who received memantine maintained better cognitive functions than patients who had placebo [5].

In vivo murine experiments suggested that the sideeffects of radiotherapy to normal brain tissue is secondary to the induction of global vascular damages in the form of devascularization, gliosis, demyelination and white matter necrosis [6]. A preliminary clinical study suggested that Dynamic Contrast-Enhanced MRI (DCE-MRI) could detect increased permeability of the BBB and bloodtumor-barrier at the completion of 60Gy of fractioned radiotherapy for the treatment of glioma [7]. Similarly, it has been suggested that DCE-MRI could be used to assess the efficacy of focused ultrasound in disrupting the BBB [8].

The current pilot study aims at determining whether
DCE-MRI is able to detect and measure changes in vascular permeability during the first 6 months following WBRT (37.5 Gy in 15 fractions) in patients with brain metastasis, and whether vascular changes in normal appearing white matter (NAWM) are associated with neurocognitive function and/or memantine use.

\section{RESULTS}

Our patient population was composed of a heterogeneous group of patients with different cancer histology and who had received various local treatments prior to WBRT (see Table 1). Most patients had lung ( $n=$ 7 ) or breast cancers $(n=4)$. Twelve patients had unresected tumor(s) present in the brain at the time of WBRT. The median overall survival from WBRT initiation was 9.75 (0-33.6) months. Seven patients received memantine, while 7 patients received either placebo or neither.

Compared to $\mathrm{K}^{\text {trans }}$ (Figure 1), AUC measurements of tumor and NAWM provided more consistent ( $\mathrm{K}^{\text {trans: }}$ 12\% failed model fitting; AUC: no missing data) and reproducible values. Mean coefficients of variation of $\mathrm{K}^{\text {trans }}$ and AUC measurements were similar in tumors (ANOVA $p=0.673)$. In NAWM, the mean coefficients of variation of AUC was significantly less (ANOVA $p=0.012$ ) than for $\mathrm{K}^{\text {trans }}$. As both measurements are recommended endpoints for the assessments and reporting of MRI oncology trials [9], we proceeded to use AUC for subsequent analysis.

We observed a trend to suggest increased AUC of tumors $(p=0.09)$ and NAWM $(p=0.06) 6$ months 


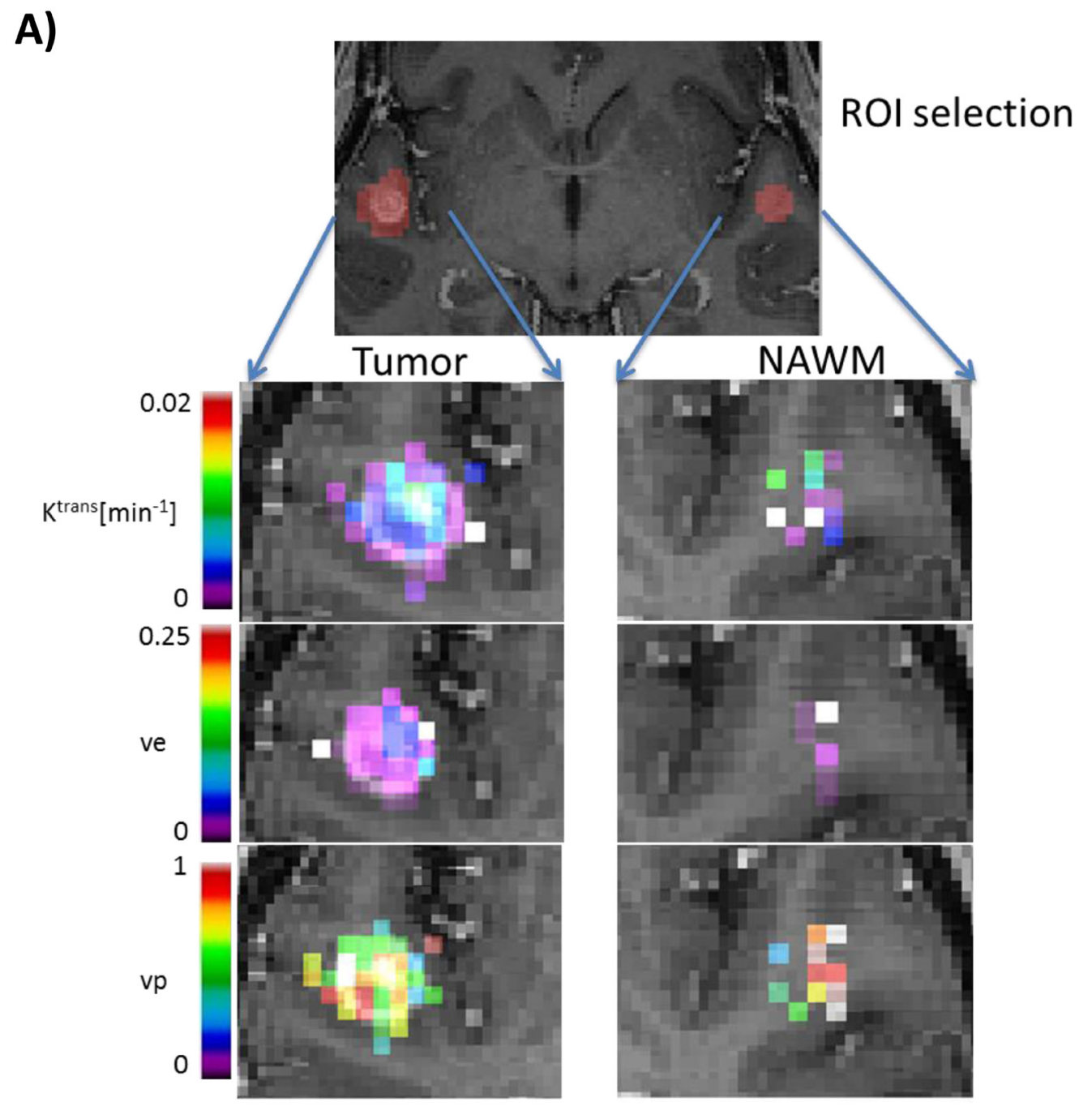

B)

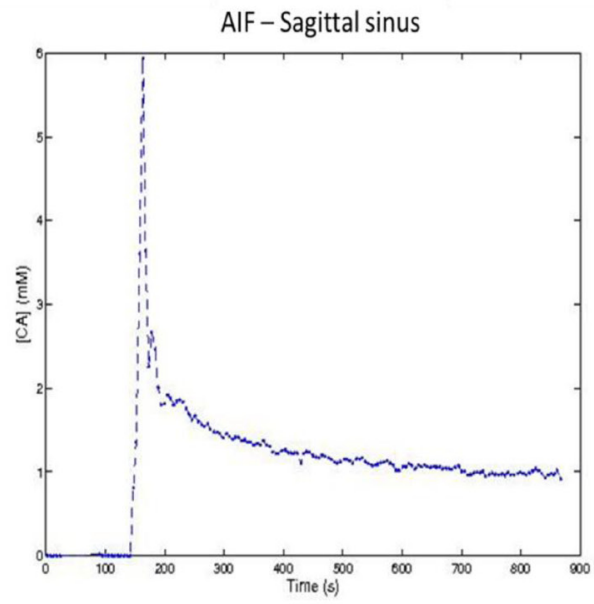

C)

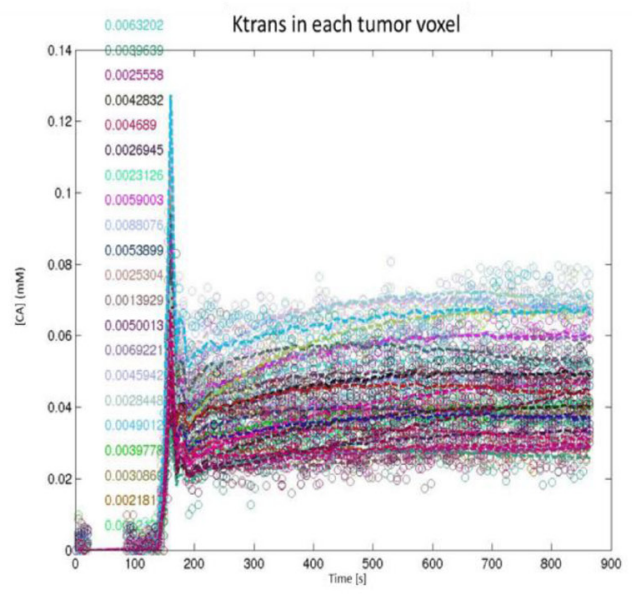

Figure 1: Sample parametric maps and time course of a tumor. Using the Tofts and Kermode modeling of DCE-MRI data, A. parametric maps of Ktrans, ve and vp were obtained for Tumor and normal appearing white matter (NAWM). Note that when the fit did not converge (Ra2 $<50 \%$ ), all the parameters are set to 0 . This was the case for many voxels within the region of interest (ROI) defined as NAWM. Ktrans: transfer constant; ve: extravascular extracellular space (EES) fractional volume; vp: blood plasma volume. A sample time course of the constrast agent concentration [CA] within B. the sagittal sinus (AIF) and $\mathbf{C}$. each Tumor voxel from Figure $1 \mathrm{~A}$. 
following WBRT initiation (Figure 2). We found that patients receiving memantine had significantly ( $p=$ 0.01 ) less NAWM AUC changes following radiotherapy than those who received placebo (Figure 3 ). When the 12 RTOG 0614 patients were analyzed separately, the memantine arm maintained a trend $(p=0.03)$ in the reduction in NAWM AUC changes following radiation as compared to the placebo arm.

Consistent with the overall results from RTOG 0614, patients on memantine retained better cognitive functions (COWA $p=0.03$ ) than those on placebo (Figure 4). HVLT $(p=0.10)$ measures were trending towards improvements in the memantine group as well. The cognitive results and their statistical significance remained the same when the analyses were repeated using RTOG 0614 patients only. QOL data were not compared as only 4 patients who had sequential MRIs completed more than 1 QOL questionnaire. Using blood samples collected prior to each MRIs, we explored the association between CEC and DCE-MRI AUC changes (Supplementary Figure 2). No association was observed between the level of CEC and DCE-MRI changes, time from radiotherapy or memantine use.

\section{DISCUSSION}

In comparison to previous trials that examined the ability of DCE-MRI to detect BBB permeability changes following high-dose radiotherapy targeted at tumors, the current pilot study demonstrated that modest dose WBRT increases the vascular permeability in normal brain tissue (mean NAWM AUC increase from baseline: 1.9 fold; $p$ $=0.06)$. Using an $\alpha / \beta=2 \mathrm{~Gy}$ for late glial cell toxicities [10], the mean normalized $2 \mathrm{~Gy}$ equivalent biologically effective dose (nBED 2/2) of 37.5Gy given in 15 fractions is $\mathrm{nBED} 2 / 2=42.2 \mathrm{~Gy}$. Our observation corroborated a previous publication that suggested that neurovascular permeability was increased following a threshold dose of 20Gy given in 30 fractions (nBED2/2 = 13.35Gy) [7]. Although the authors also observed a recovery in the vascular permeability of the brain parenchyma 6 months after radiotherapy, our placebo patients did not show any recovery in their NAWM AUC within the first 6 months after WBRT (Figure 3), suggesting a potential dose related effect to brain vasculature damage and recovery. Similarly, tumor contrast uptake increased over the first 6 months from WBRT.

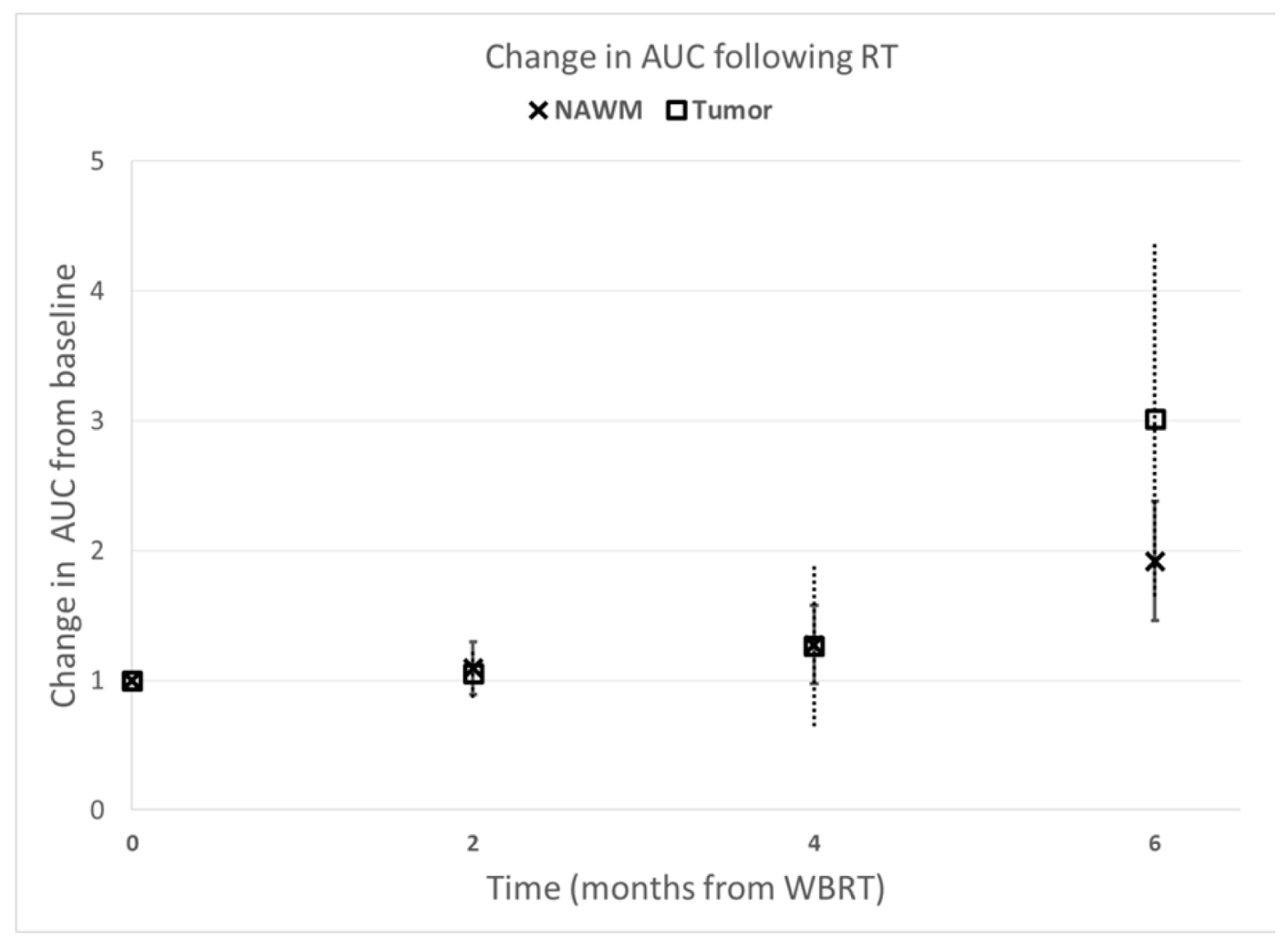

Figure 2: Tumor and normal tissue vascular permeability changes following brain irradiation. Contrast uptake following brain irradiation. Increase in contrast uptake (Area Under the uptake Curve (AUC)) of normal appearing white matter (NAWM) and tumor following whole brain irradiation (WBRT). Data were normalized to the AUC at baseline, prior to beginning WBRT. Error bars represent the standard error of the means. 
The detrimental effect of brain radiotherapy is well documented, particularly in studies involving the treatment of childhood cancers [11]. In childhood cancer survivors, the neurocognitive morbidity of radiotherapy is associated with higher radiation dose, larger volume of irradiation, and younger age at the time of treatment. In adult patients, multiple studies examined the consequences of prophylactic cranial irradiation to patient reported QOL. Using EORTC QOL questionnaires, Slotman et al. and Le Pechoux et al. did not detect significant differences in self-reported cognitive functions between patients who received $25 \mathrm{~Gy} / 10$ fractions $(\mathrm{nBED} 2 / 2=$ $28.13 \mathrm{~Gy}) v s$. observation [12] or $25 \mathrm{~Gy} / 10$ fractions $v s$. 36Gy/18-24 fractions [13] (nBED2/2 = 31.5-36Gy), respectively. However, when outcomes were evaluated using standardized neurocognitive tests, Welzel et al. documented acute cognitive dysfunctions soon after WBRT is initiated that remained significant 2-4 weeks after the completion of WBRT [14]. Wolfson et al. similarly found radiation dose and age dependence in the development of neurocognitive defects [15]. Finally, Sun et al. found that in comparison with patients who were observed, patients who received prophylactic cranial irradiation (30Gy in 15 fractions; nBED2/2 = 30Gy) developed significant declines in immediate and delayed recall beginning at 3 months post-WBRT, with mild and incomplete recovery in the following months [16].

The mechanism by which radiation induces brain injury is likely secondary to injuries to multiple cell types, including vascular [17] and parenchymal cells such as neuronal and glial cells [6]. Radiation may induce vascular damage, which leads to ischemia that subsequently results in NMDA receptor stimulation and excitotoxicity. Memantine is a NMDA receptor competitive antagonist that is approved for the treatment of Alzheimer's disease and has shown mixed efficacies in the treatment of vascular dementia. The RTOG 0614 study demonstrated that starting at 8 weeks from WBRT, patients on memantine had less cognitive decline than the placebo cohort [5]. In our study, we observed that patients on memantine were also protected from radiation induced NAWM vasculature permeabilization (Figures 3-4). Our findings were in accordance with another preliminary study that suggested that DCE-MRI could be an imaging biomarker of changes in neurovasculature permeability and increased permeability following radiotherapy potentially predicted for the development of neurocognitive dysfunction following radiotherapy [7]. Similarly, DCE-MRI may be used to detect subtle BBB permeability changes in early neurodegenerative diseases such as Alzheimer's disease [18-20]. Furthermore, a pre-clinical study suggested that memantine reduced brain edema, BBB permeability, infarct volume and neurological deficits when it is given 15 min following middle cerebral artery occlusion in rats [21]. These prior studies corroborate with our results that suggested that memantine not only protected neurons from radiation-induced excitotoxicity, but might also protect the cerebral vasculature from radiation damages.

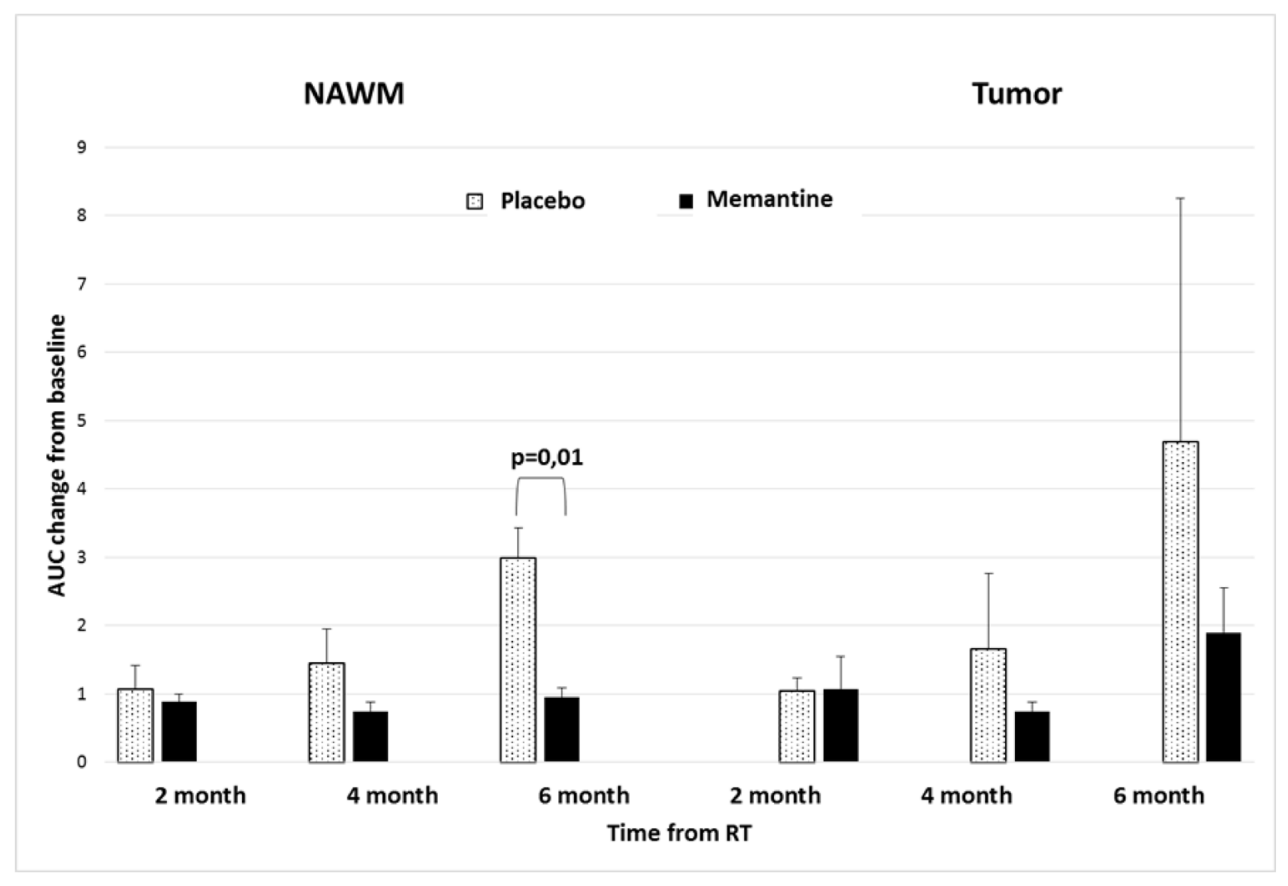

Figure 3: Tissue contrast uptake after irradiation in relation to memantine use. Contrast uptake (AUC) of normal appearing white matter (NAWM) and tumor in patients on placebo and memantine. NAWM of patients receiving memantine have reduced AUC changes following radiotherapy $(p=0.01)$ in comparison to patients receiving placebo. Error bars represent the standard error of the means. 
As shown in Supplementary Figure 3 and 4, the genes (GRIN1, GRIN2A, GRIN2B, GRIN2C, and GRIN2D) and protein of NMDA receptors subunits are widely expressed in different normal tissues and cells, including endothelial cells. Preclinical studies suggested that stimulation of cerebral endothelial cell NMDA receptors could induce vasodilation of brain arteries or excitotoxicity and breakdown of the BBB [22]. Therefore, the neuroprotective effects of memantine might be secondary to the combined reduction in neuronal and endothelial cell deaths from irradiation.

In our study, there was no significant $(p=0.20)$ difference in overall survival of patients on memantine or placebo. There was no significant correlation between the vascular permeability of tumors or NAWM at any time point with overall survival. The majority $(78.6 \%)$ of our patients had lung or breast cancers, which is in keeping with the patient population in RTOG 0614 (84.6\% lung and breast cancers). Results from the RTOG 0614 [5] trial demonstrated that patients who received memantine $(n=$ $256)$ or placebo $(n=252)$ had similar median progression free survival (4.7 vs. 5.5 months; $p=0.27$ ) and overall survival (6.7 vs. 7.8 months; $p=0.28)$. There was also no additional toxicity observed among patients who received memantine $v s$. placebo. Therefore, use of memantine and its neurovascular protective effect did not seem to affect

\section{Time from WBRT}

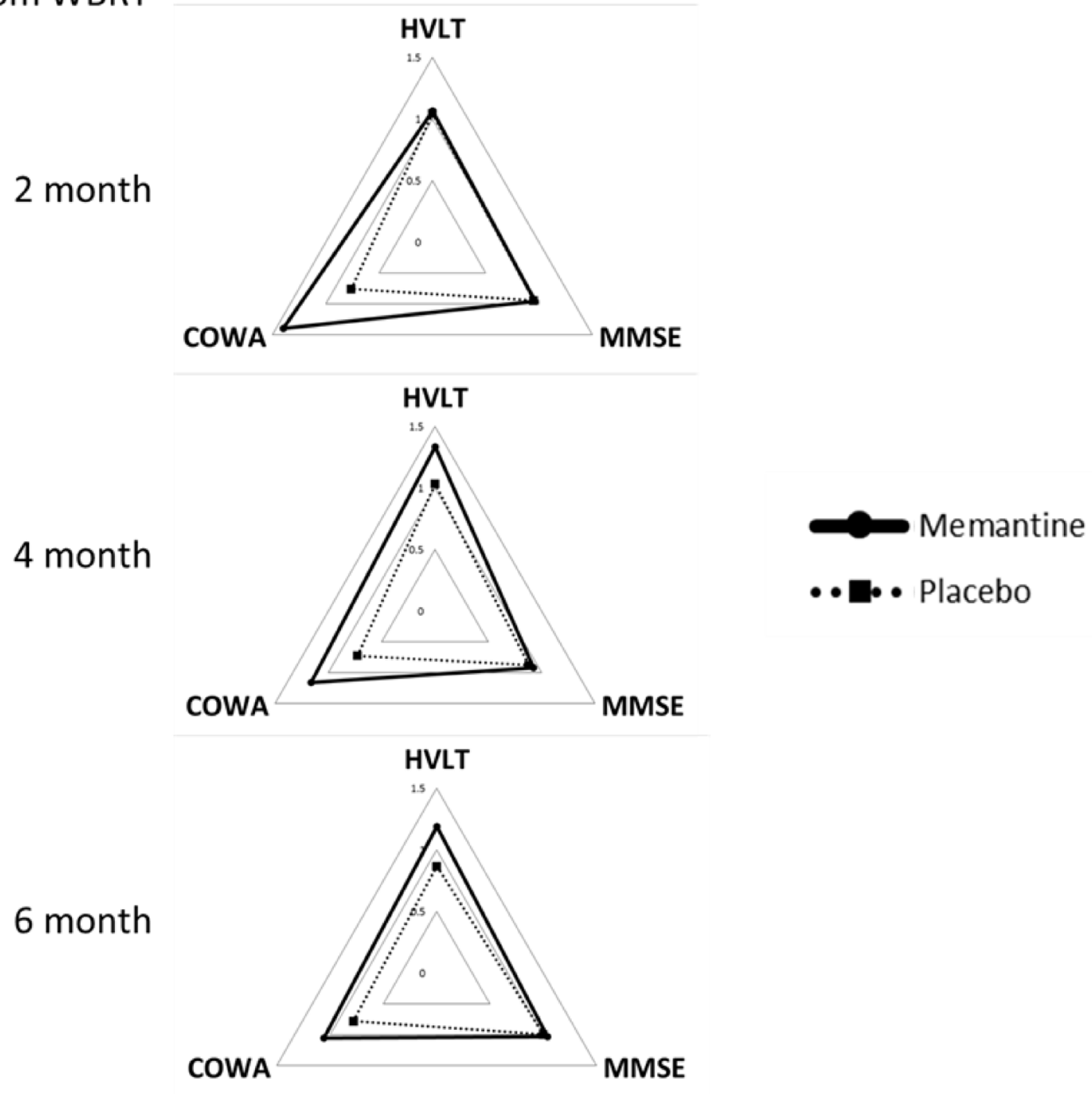

Figure 4: Neurocognitive functions following brain irradiation. Neurocognitive functions following brain irradiation. Neurocognitive functions (HVTL, MMSE, and COWA) of patients at 2, 4 and 6 months from receiving whole brain radiotherapy (WBRT) with memantine (solid) or placebo (dotted). Data were normalized to individual patients' baseline values. Higher values represent better quality of life or neurocognitive functions; values $>1$ represents an improvement from baseline. 
tumor control, patient survival or toxicities.

Due to the small number of patients in this pilot study, our results need to be confirmed using a larger cohort of patients. Also, data from this small patient cohort did not allow us to determine whether surgery and/ or radiosurgery influenced the vascular permeability of tumors. Our imaging methodologies could be modified to study the radiotherapy effects to specific brain structures, such as the hippocampus by increasing the spatial resolution of the DCE-MRI acquisition at this structure and by using a power injector. As the gene and protein expressions of different NMDA receptors vary between brain structures (Supplementary Figure 3 and 4), the benefit of memantine may differ between tissues and cell types [23, 24]. Furthermore, as many long-term toxicities secondary to radiation therapy are related to the devascularization of normal tissues, the use of memantine as an adjunct to radiotherapy may reduce extra-cerebral endothelial cell injuries and the rate of chronic radiation side-effects in a broad range of patients.

In conclusion, DCE-MRI represents an established functional imaging method employed in evaluating vascular permeability changes in tissues and tumors. Radiation therapy induces vasculature damages and many long-term toxicities are related to the de-vascularization of normal tissues. Our pilot study suggests that DCE-MRI could detect BBB vascular damages secondary to WBRT and could be used as a biomarker of neurocognitive dysfunction. Our study also suggested that memantine reduced WBRT damages to neurons and endothelial cells. Future studies should aim at confirming the usefulness of DCE-MRI as a clinical imaging biomarker of tissue toxicity from radiotherapy. The use of memantine as a potential radioprotector for vascular tissues may be explored to reduce radiation induced side-effects.

\section{PATIENTS AND METHODS}

\section{Patients}

Fourteen adult patients with a pathologically proven diagnosis of solid malignancy within 5 years of registration and with brain metastases visible on contrastenhanced MRI were recruited to the study. One patient died before WBRT was started. Eligibility criteria included a Karnofsky performance status of $\geq 70$, stable systemic disease in the 3 months prior to study entry, serum creatinine $\leq 3 \mathrm{mg} / \mathrm{dL}$, creatinine clearance $\geq 30 \mathrm{~mL} / \mathrm{min}$, total bilirubin $\leq 2.5 \mathrm{mg} / \mathrm{dL}$, blood urea nitrogen (BUN) $\leq 20 \mathrm{mg} / \mathrm{dL}$, Mini Mental State Exam (MMSE) score $\geq$ 18 within 28 days of study entry, no current alcohol or drug abuse, no chronic use of benzodiazepines, and no severe active comorbidity. Patients could have received prior therapy for brain metastasis, including radiosurgery and surgical resection (but no prior cranial external beam radiotherapy). Patients receiving systemic therapy were eligible if such therapy was given $>14$ days prior to study entry, and they could not receive chemotherapy for at least 14 days after completing radiotherapy. Table 1 summarizes the patients' characteristics. The study was approved by the McGill University Health Centre Research Ethics Board. All patients provided written informed consent.

\section{Treatment}

All 12 patients concurrently randomized on RTOG 0614 were treated with WBRT consisting of 37.5 Gy in 15 fractions, delivered using 2 parallel-opposed fields with or without subfields. Of the 2 patients not on RTOG 0614, patient \#3 received 30 Gy in 12 fractions and patient \#10 received 37.5 Gy in 15 fractions, delivered using 2 parallel-opposed fields with or without subfields (Table 1). Patients enrolled in the RTOG 0614 trial were randomly assigned to received placebo $(n=5)$ or memantine $(n=7)$ at the initiation of WBRT. Patient on memantine received $5 \mathrm{mg}$ on week 1 , increasing by $5 \mathrm{mg}$ every week until the target dose of $10 \mathrm{mg}$ twice daily, and was then maintained at this dose for a total of 24 weeks [5]. Patients not on the RTOG0614 did not receive placebo or memantine; for the purpose of analysis, data from these patients were combined with those who received placebo. Following the completion and publication of final data from the RTOG 0614 study, we obtained permission from the RTOG to unblind the treatment (placebo or memantine) that patients received during and after WBRT. One of the 14 patients died prior to receiving the first fraction of WBRT.

\section{Imaging}

Patients underwent MRI's prior to WBRT (within 28 days from start of WBRT), then at 8-weeks, 16-weeks and 24-weeks after the initiation of WBRT.

Timing and MRI sequence acquisition were modified from the methods described by Roberts et al. [25]. All images were acquired on a Siemens 3T Tim Trio with a maximum gradient strength of $40 \mathrm{mT} / \mathrm{m}$ and a slew rate of $200 \mathrm{mT} / \mathrm{m} / \mathrm{ms}$, using a Siemens 32-channel head coil.

Briefly, a sagittal T2-weighted scout scan was obtained to plan a whole brain T1-weigthed MPRAGE (matrix: 256x256x176, $1 \mathrm{~mm}^{3}$ isotropic resolution, TR/TE $=2.3 \mathrm{~s} / 3.4 \mathrm{~ms}, \mathrm{FA}=9^{\circ}$ ), localize the tumor, and plan the dynamic series. For DCE-MRI, a 3D-FLASH sequence was obtained with the following parameters: TR/TE = 4.4/1.5ms; flip angle: $25^{\circ}$; matrix: 128 x 128, 22 slices; resolution: $2 \times 2 \times 6 \mathrm{~mm}^{3}$; $\mathrm{BW}=1775 \mathrm{~Hz} / \mathrm{px}$; GRAPPA factor 2 . This $3 \mathrm{D}$ data set was acquired every 6 secs, starting before intravenous bolus administration of a single dose of contrast material $\left(0.1 \mathrm{mmol} / \mathrm{kg}\right.$ Gadovist ${ }^{\circledR}$, Bayer 
Inc.) and continuing for the subsequent 15 minutes, for a total of 155 dynamic images. The contrast agent was administered manually as a bolus injection and chased with $30 \mathrm{ml}$ of saline. At the completion of 15 minutes of DCE-MRI, a second, post-contrast MPRAGE, matched to the first acquisition, was acquired. All kinetic images and post-contrast MPRAGE were registered to the initial pre-contrast MPRAGE using the MINC software tools (http://packages.bic.mni.mcgill.ca/tgz). Sixparameter linear registration using mutual information was computed between the first dynamic image and the MPRAGE anatomical. Then each image from the dynamic series was registered to the first image using a 6-parameter linear transformation using cross-correlation. The transformations from these two linear registrations were concatenated and applied to each dynamic image so that all frames were registered to the high resolution anatomical.

3D-FLASH images were acquired for purposes of T1-mapping using the DESPOT1 technique (FA = $2^{\circ} / 10^{\circ}, 128 \times 128 \times 22$ matrix, resolution $2 \times 2 \times 6 \mathrm{~mm} 3$ ) [26] (Supplementary Figure 1). The T1-weighted time series was used together with the equilibrium magnetization (M0) map calculated with DESPOT1 to generate quantitative T1 4D volumes and were subsequently converted to concentration using the pre-contrast T1 (T10) maps and contrast agent relaxivity in the plasma (values at $3 \mathrm{~T}, 37^{\circ} \mathrm{C}, \mathrm{pH}=7$, in plasma: $\mathrm{r} 1(\mathrm{Gd}-\mathrm{BT}-\mathrm{DO} 3 \mathrm{~A})=5.0 \pm 0.3$ mM-1s-1 [27-29]).

All regions of interests (ROI) (tumor, NAWM, sagittal sinus) were manually segmented on the postcontrast MPRAGE by PW. The area of tumor was defined as the contrast enhancing volumes (using the pre and post-injection MPRAGE scans) if the tumor was not resected. If multiple tumors were present, each tumor was segmented as an individual ROI. In the case of resected tumors, any contrast-enhancement surrounding the tumor cavity was contoured. A parenchymal area of the brain contralateral to the tumor, without contrast enhancement on post-injection MPRAGE scans, was considered as NAWM. A segment of the superior sagittal sinus was contoured using contrast-enhanced images (3D-FLASH and MPRAGE sequences) and used for measuring the vascular time course of the contrast agent concentration. Although signals from the sagittal sinus are not arterial, the large size of this vein reduces experimental/analytical partial volume effects. This methodology has been shown to be highly reproducible $[30,31]$ and a good surrogate for the arterial input function $[32,33]$.

Concentrations over time were computed using a Matlab (Mathworks, Natick, MA) program developed inhouse, and the sagittal sinus ROI was used to define the blood input function. The signal intensity changes (signal intensity after contrast agent administration minus that before contrast agent administration) were calculated for the ROIs for each post-injection time point, and their time courses were used for subsequent kinetic analyses.

Vascular permeability was evaluated using the semiquantitative Initial Area Under the Curve (AUC) Blood Adjusted method [34, 35], which was calculated as the area under the ROI signal intensity curve from the time of injection to 90 seconds post-injection, divided by the area under the blood concentration curve for the same period of time. This methodology provided simple and robust measurements that did not require model fitting and has been recommended as a primary measure in MRI trials of oncology therapeutics [9]. We also explored the traditional kinetic modeling methods described by Tofts et al [36]. Specifically, a bidirectional 3-compartment model based on the equations of Tofts and Kermode [36], which yielded estimates of fractional tissue blood volume (in milliliters per cubic centimeters), plasma volume, and microvascular permeability, which was expressed as the transendothelial transfer constant, $\mathrm{K}^{\text {trans }}$, was also evaluated. An example of parametric maps of $\mathrm{K}^{\text {trans }}$, ve and $\mathrm{vp}$ and the corresponding $\mathrm{K}^{\text {trans }}$ of tumor voxels obtained from the Tofts and Kermode modeling are shown in Figure 1.

\section{Quality of life and neurocognitive assessments}

At each MRI time point, cognitive, quality-of-life (QOL) assessment and blood samples were collected according to the patient's ability to tolerate the exams. Cognitive assessments were done using the MMSE, Hopkins Verbal Learning Test (HVLT), and Controlled Oral Word Association Test (COWA). QOL assessments were made using validated questionnaires: FACT-G and FACT-Br.

\section{Circulating endothelial cell assessments}

Circulating endothelial cells (CEC) were measured as per the protocol described by Duda et al. [37]. Briefly, patient blood was collected prior to MRI imaging using tubes containing EDTA. Fluorescently labelled antibodies were purchased from BD Pharmingen: CD31-FITC, CD34-APC, CD133-PE, CD45-PerCP, Fluorescently labeled isotype-matched IgG1 antibodies, and VEGFR2 (KDR)-PE. Cells from the buffy coat were fixed, labeled using the above listed antibodies, then sorted using the FACSCalibur flow cytometer (Becton Dickinson).

\section{Analysis plan}

This study was a prospective pilot study with the aim of recruiting up to 15 patients in order to determine the feasibility and ability of DCE-MRI in detecting vascular permeability changes secondary to WBRT. With no prior evidence of DCE-MRI's capacity to detect WBRT-related NAWM neurovascular changes at the time 
of the trial design, the study was not pre-designed with statistical power estimations. As Cao et al. observed an initial increase in vascular permeability followed by a gradual recovery after the completion of radiotherapy [7], a linear change in vascular permeability over time could not be inferred; hence linear regression and slope analysis might not be appropriate for our data. Therefore, the analysis consisted of comparing the vascular permeability in tumor and NAWM before $v s$. after WBRT (3 time points within 6 months from the start of WBRT) using the paired student $t$-test to compare baseline data with 2, 4 and 6 months MRI data. Coefficient of variations of DCE-MRI measurements ( $\mathrm{K}^{\text {trans }}$ and AUC) were calculated $\left(C_{v}=\right.$ Standard deviation/Mean) and compared using ANOVA. Comparison between placebo and memantine for MRI and neurocognitive results were made using the student $t$-test at each time point. Changes in CEC levels were analyzed using the spearman correlation test to determine its association with MRI data. All analyses were twosided and a $P$-value of $<0.05$ is considered as statistically significant. The Bonferroni correction was applied to the change in vascular permeability analyses of 3 different time points $(2,4$ and 6 months) such that a $P$-value of $<$ 0.017 is considered statistically significant.

\section{CONFLICTS OF INTEREST}

The authors declare that they have no conflicts of interest to disclose.

\section{GRANT SUPPORT}

Funding was provided by the Cedars Cancer Institute H. R. Shibata Cedars Scholarship Award to PW and CM.

Gadovist $^{\circledR}$ was provided in kind by Bayer Inc.

\section{Editorial note}

This paper has been accepted based in part on peerreview conducted by another journal and the authors' response and revisions as well as expedited peer-review in Oncotarget.

\section{REFERENCES}

1. Soliman H, Das S, Larson DA and Sahgal A. Stereotactic radiosurgery (SRS) in the modern management of patients with brain metastases. Oncotarget. 2016.

2. Lin $X$ and DeAngelis LM. Treatment of Brain Metastases. Journal of clinical oncology. 2015; 33(30):3475-3484.

3. Tsao MN, Rades D, Wirth A, Lo SS, Danielson BL, Gaspar LE, Sperduto PW, Vogelbaum MA, Radawski JD, Wang JZ, Gillin MT, Mohideen N, Hahn CA and Chang EL. Radiotherapeutic and surgical management for newly diagnosed brain metastasis(es): An American Society for Radiation Oncology evidence-based guideline. Pract Radiat Oncol. 2012; 2(3):210-225.

4. Owonikoko TK, Arbiser J, Zelnak A, Shu HK, Shim H, Robin AM, Kalkanis SN, Whitsett TG, Salhia B, Tran NL, Ryken T, Moore MK, Egan KM and Olson JJ. Current approaches to the treatment of metastatic brain tumours. Nature reviews Clinical oncology. 2014; 11(4):203-222.

5. Brown PD, Pugh S, Laack NN, Wefel JS, Khuntia D, Meyers C, Choucair A, Fox S, Suh JH, Roberge D, Kavadi V, Bentzen SM, Mehta MP, Watkins-Bruner D and Radiation Therapy Oncology G. Memantine for the prevention of cognitive dysfunction in patients receiving whole-brain radiotherapy: a randomized, doubleblind, placebo-controlled trial. Neuro-oncology. 2013; 15(10):1429-1437.

6. Belka C, Budach W, Kortmann RD and Bamberg M. Radiation induced CNS toxicity--molecular and cellular mechanisms. British journal of cancer. 2001; 85(9):12331239.

7. Cao Y, Tsien CI, Sundgren PC, Nagesh V, Normolle D, Buchtel H, Junck L and Lawrence TS. Dynamic contrastenhanced magnetic resonance imaging as a biomarker for prediction of radiation-induced neurocognitive dysfunction. Clinical cancer research. 2009; 15(5):1747-1754.

8. Vlachos F, Tung YS and Konofagou E. Permeability dependence study of the focused ultrasound-induced bloodbrain barrier opening at distinct pressures and microbubble diameters using DCE-MRI. Magnetic resonance in medicine. 2011; 66(3):821-830.

9. Leach MO, Brindle KM, Evelhoch JL, Griffiths JR, Horsman MR, Jackson A, Jayson GC, Judson IR, Knopp MV, Maxwell RJ, McIntyre D, Padhani AR, Price P, Rathbone R, Rustin GJ, Tofts PS, et al. The assessment of antiangiogenic and antivascular therapies in early-stage clinical trials using magnetic resonance imaging: issues and recommendations. British journal of cancer. 2005; 92(9):1599-1610.

10. Fowler JF. The linear-quadratic formula and progress in fractionated radiotherapy. The British journal of radiology. 1989; 62(740):679-694.

11. Padovani L, Andre N, Constine LS and Muracciole X. Neurocognitive function after radiotherapy for paediatric brain tumours. Nature reviews Neurology. 2012; 8(10):578588.

12. Slotman BJ, Mauer ME, Bottomley A, Faivre-Finn C, Kramer GW, Rankin EM, Snee M, Hatton M, Postmus PE, Collette L and Senan S. Prophylactic cranial irradiation in extensive disease small-cell lung cancer: short-term healthrelated quality of life and patient reported symptoms: results of an international Phase III randomized controlled trial by the EORTC Radiation Oncology and Lung Cancer Groups. Journal of clinical oncology. 2009; 27(1):78-84.

13. Le Pechoux C, Laplanche A, Faivre-Finn C, Ciuleanu T, Wanders R, Lerouge D, Keus R, Hatton M, Videtic 
GM, Senan S, Wolfson A, Jones R, Arriagada R, Quoix E, Dunant A and Prophylactic Cranial Irradiation Collaborative G. Clinical neurological outcome and quality of life among patients with limited small-cell cancer treated with two different doses of prophylactic cranial irradiation in the intergroup phase III trial (PCI99-01, EORTC 2200308004, RTOG 0212 and IFCT 99-01). Annals of oncology. 2011; 22(5):1154-1163.

14. Welzel G, Fleckenstein K, Schaefer J, Hermann B, KrausTiefenbacher U, Mai SK and Wenz F. Memory function before and after whole brain radiotherapy in patients with and without brain metastases. International journal of radiation oncology, biology, physics. 2008; 72(5):13111318.

15. Wolfson AH, Bae K, Komaki R, Meyers C, Movsas B, Le Pechoux C, Werner-Wasik M, Videtic GM, Garces YI and Choy H. Primary analysis of a phase II randomized trial Radiation Therapy Oncology Group (RTOG) 0212: impact of different total doses and schedules of prophylactic cranial irradiation on chronic neurotoxicity and quality of life for patients with limited-disease small-cell lung cancer. International journal of radiation oncology, biology, physics. 2011; 81(1):77-84

16. Sun A, Bae K, Gore EM, Movsas B, Wong SJ, Meyers CA, Bonner JA, Schild SE, Gaspar LE, Bogart JA, WernerWasik M and Choy H. Phase III trial of prophylactic cranial irradiation compared with observation in patients with locally advanced non-small-cell lung cancer: neurocognitive and quality-of-life analysis. Journal of clinical oncology. 2011; 29(3):279-286.

17. Stewart FA, Hoving S and Russell NS. Vascular damage as an underlying mechanism of cardiac and cerebral toxicity in irradiated cancer patients. Radiation research. 2010; 174(6):865-869.

18. Barnes SR, Ng TS, Montagne A, Law M, Zlokovic BV and Jacobs RE. Optimal acquisition and modeling parameters for accurate assessment of low $\mathrm{K}$ blood-brain barrier permeability using dynamic contrast-enhanced MRI. Magnetic resonance in medicine. 2015.

19. Starr JM, Farrall AJ, Armitage P, McGurn B and Wardlaw J. Blood-brain barrier permeability in Alzheimer's disease: a case-control MRI study. Psychiatry Res. 2009; 171(3):232-241.

20. Wang H, Golob EJ and Su MY. Vascular volume and blood-brain barrier permeability measured by dynamic contrast enhanced MRI in hippocampus and cerebellum of patients with MCI and normal controls. Journal of magnetic resonance imaging. 2006; 24(3):695-700.

21. Gorgulu A, Kins T, Cobanoglu S, Unal F, Izgi NI, Yanik $\mathrm{B}$ and Kucuk M. Reduction of edema and infarction by Memantine and MK-801 after focal cerebral ischaemia and reperfusion in rat. Acta Neurochir (Wien). 2000; 142(11):1287-1292.

22. Sharp CD, Hines I, Houghton J, Warren A, Jackson THt, Jawahar A, Nanda A, Elrod JW, Long A, Chi A, Minagar
A and Alexander JS. Glutamate causes a loss in human cerebral endothelial barrier integrity through activation of NMDA receptor. American journal of physiology Heart and circulatory physiology. 2003; 285(6):H2592-2598.

23. Wu C, Macleod I and Su AI. BioGPS and MyGene.info: organizing online, gene-centric information. Nucleic acids research. 2013; 41(Database issue):D561-565.

24. Uhlen M, Oksvold P, Fagerberg L, Lundberg E, Jonasson K, Forsberg M, Zwahlen M, Kampf C, Wester K, Hober $\mathrm{S}$, Wernerus $\mathrm{H}$, Bjorling $\mathrm{L}$ and Ponten F. Towards a knowledge-based Human Protein Atlas. Nature biotechnology. 2010; 28(12):1248-1250.

25. Roberts HC, Roberts TP, Brasch RC and Dillon WP. Quantitative measurement of microvascular permeability in human brain tumors achieved using dynamic contrastenhanced MR imaging: correlation with histologic grade. AJNR American journal of neuroradiology. 2000; 21(5):891-899.

26. Deoni SC, Rutt BK and Peters TM. Rapid combined T1 and T2 mapping using gradient recalled acquisition in the steady state Magn Reson Med 200349 ( 3 ): 515-526. .

27. Rohrer M, Bauer H, Mintorovitch J, Requardt $M$ and Weinmann H-J. Comparison of Magnetic Properties of MRI Contrast Media Solutions at Different Magnetic Field Strengths. Investigative Radiology. 2005; 40(11):715-724.

28. Zhu XP, Li KL, Kamaly-Asl ID, Checkley DR, Tessier JJ, Waterton JC and Jackson A. Quantification of endothelial permeability, leakage space, and blood volume in brain tumors using combined $\mathrm{T} 1$ and $\mathrm{T} 2 *$ contrast-enhanced dynamic MR imaging. Journal of magnetic resonance imaging. 2000; 11(6):575-585.

29. Zhu XP, Li KL, Waterton J, Tessier JJL, Checkley D, Jones A, Kamaly-Asl ID and Jackson A. (1999). 3D T1 Mapping by Means of Fast Field Echo Technique. 8th ISMRM Annual Meeting. (Denver, pp. 2143.

30. Lavini C and Verhoeff JJ. Reproducibility of the gadolinium concentration measurements and of the fitting parameters of the vascular input function in the superior sagittal sinus in a patient population. Magnetic resonance imaging. 2010; 28(10):1420-1430.

31. Wu Y, Wu C, Chiang C, Chen C and Chai J. (2012). Consistency of permeability measurement using arterial input function and venous output function in DCE-MRI for metastatic brain tumors. . 20th Annual Meeting of ISMRM. (Melbourne, pp. 3508).

32. Foottit C, Cron GO, Hogan MJ, Nguyen TB and Cameron I. Determination of the venous output function from MR signal phase: feasibility for quantitative DCE-MRI in human brain. Magnetic resonance in medicine. 2010; 63(3):772-781.

33. Foottit C, Cron GO, Mercier JF, Nguyen TV, Cameron I, Schweitzer ME, Sinclair J, Woulfe J, Hogan MJ and Nguyen TB. (2011). Optimizing perfusion imaging of brain tumors: Validation of venous output function used 
as a surrogate AIF. 19th Annual Meeting of ISMRM. (Montreal, pp. 2038).

34. Evelhoch JL. Key factors in the acquisition of contrast kinetic data for oncology. Journal of magnetic resonance imaging : JMRI. 1999; 10(3):254-259.

35. Port RE, Bernstein LJ, Barboriak DP, Xu L, Roberts TP and van Bruggen N. Noncompartmental kinetic analysis of DCE-MRI data from malignant tumors: Application to glioblastoma treated with bevacizumab. Magnetic resonance in medicine. 2010; 64(2):408-417.
36. Tofts PS, Brix G, Buckley DL, Evelhoch JL, Henderson E, Knopp MV, Larsson HB, Lee TY, Mayr NA, Parker GJ, Port RE, Taylor J and Weisskoff RM. Estimating kinetic parameters from dynamic contrast-enhanced T(1)-weighted MRI of a diffusable tracer: standardized quantities and symbols. Journal of magnetic resonance imaging. 1999; 10(3):223-232.

37. Duda DG, Cohen KS, Scadden DT and Jain RK. A protocol for phenotypic detection and enumeration of circulating endothelial cells and circulating progenitor cells in human blood. Nature protocols. 2007; 2(4):805-810. 